\title{
Isotopic Dependence of Nuclear Charge Radii and Pairing Energies
}

\author{
U. Regge and D. Zawischa \\ Institut für Theoretische Physik, Universität Hannover, West Germany \\ (Received 5 February 1988)
}

\begin{abstract}
With a residual interaction consisting of density-independent two- and three-body parts, we obtain within Hartree-Fock-Bogolyubov theory at the same time good results on the odd-even staggering of charge radii and on trends of the pairing energies. We present results for $\mathrm{Sn}$ and some isotopic chains in the Ba region.

PACS numbers: $21.10 . \mathrm{Dr}, 21.10 . \mathrm{Ft}, 21.30 .+\mathrm{y}, 27.60 .+\mathrm{j}$
\end{abstract}

In a recent paper ${ }^{1}$ it has been shown that the staggering of nuclear charge radii can easily be explained if one assumes a suitable three- or four-body part in the effective residual interaction. Here by a more rigorous treatment using a repulsive three-body contact force we reproduce systematic trends of the pairing energies ${ }^{2}$ and obtain in addition improved results on the charge radii.

For the derivation of our equations we start with the Hamiltonian

$$
H=\sum_{i} \epsilon_{i} a_{i}^{\dagger} a_{i}+\frac{1}{2} \sum_{i j k l} V_{i j k l}^{(2)} a_{i}^{\dagger} a_{j}^{\dagger} a_{l} a_{k}+\frac{1}{6} \sum_{i j k l m n} V_{i j k l m n}^{(3)} a_{i}^{\dagger} a_{j}^{\dagger} a_{k}^{\dagger} a_{n} a_{m} l
$$

where $V_{i j k l}^{(2)}$ and $V_{i j k l m n}^{(3)}$ are not antisymmetrized. With the employment of the method of Green's functions, in the resulting hierarchy of equations the $n$-particle function is connected with the $(n+1)$-particle function by the two-body interaction, and with the $(n+2)$-particle function by $V^{(3)}$. Following Gorkov ${ }^{3}$ we introduce the anomalous Green's functions $F, F^{\dagger}$ which obey equations of motion analogous to those for $G$, and factorize $G^{(2)}$ as well as $G^{(3)}$. After Fourier transformation, we finally arrive at equations of standard Hartree-Fock-Bogolyubov form

$$
\begin{aligned}
& G_{i j}(\mu+\omega)=G_{i j}^{0}(\mu+\omega)+\sum_{k l} G_{i k}^{0}(\mu+\omega)\left[\Gamma_{k l} G_{l j}(\mu+\omega)-\Delta_{k l} F_{l j}^{\dagger}(\mu+\omega)\right], \\
& F_{i j}^{\dagger}(\mu+\omega)=\sum_{k l} G_{i k}^{0}(\mu-\omega)\left[\Gamma_{k l}^{*} F_{l j}^{\dagger}(\mu+\omega)-\Delta_{k l}^{*} G_{l j}(\mu+\omega)\right] .
\end{aligned}
$$

The chemical potential $\mu$ is fixed by average particle-number conservation. The quantities $\Gamma$ and $\Delta$ are given by

$$
\begin{aligned}
\Gamma_{i j} & =\sum_{k l} \bar{V}_{i l j k}^{(2)} \rho_{k l}+\frac{1}{2} \sum_{k l m n} \bar{V}_{i l n j k m}^{(3)} \rho_{m n} \rho_{k l}+\frac{1}{4} \sum_{k l m n} \bar{V}_{i k l j m n}^{(3)} \phi_{m n} \phi_{k l}^{*}, \\
\Delta_{i j} & =\frac{1}{2} \sum_{k l} \bar{V}_{i j k l}^{(2)} \phi_{k l}+\frac{1}{2} \sum_{k l m n} \bar{V}_{i j n k l m}^{(3)} \rho_{m n} \phi_{k l},
\end{aligned}
$$

with $\rho_{i j}=(2 \pi i)^{-1} \int d \omega G_{i j}(\omega)$ being the normal and $\phi_{i j}=(2 \pi i)^{-1} \int d \omega F_{i j}(\omega)$ the anomalous (or pairing) density, and $\bar{V}_{i j k l}^{(2)}$ and $\bar{V}_{i j k l m n}^{(3)}$ denoting fully antisymmetrized matrix elements.

Equations (2) are solved in two steps. We write (in an obvious shorthand notation) $\Gamma=\Gamma^{0}+\delta \Gamma$ and obtain by iteration and resummation the equations

$$
\begin{aligned}
& G=\tilde{G}^{0}+\tilde{G}^{0} \delta \Gamma G-\tilde{G}^{0} \Delta F^{\dagger}, \\
& F^{\dagger}=\tilde{G}^{0} \delta \Gamma^{*} F^{\dagger}-\tilde{G}^{0}-\Delta^{*} G,
\end{aligned}
$$

where the subscript - indicates the negative-energy argument. $\tilde{G}^{0}$ and $\Gamma^{0}$ are determined by

$$
\begin{aligned}
& \tilde{G}^{0}=G^{0}+G^{0} \Gamma^{0} \tilde{G}^{0}, \\
& \Gamma^{0}=\bar{V}^{(2)} \rho^{0}+\frac{1}{2} \bar{V}^{(3)} \rho^{0} \rho^{0}, \quad \rho_{i j}^{0}=\int \frac{d \omega}{2 \pi i} \tilde{G}_{i j}^{0}(\omega) .
\end{aligned}
$$

Equations (6) represent a self-consistent Hartree-Fock problem and may be solved by iterative diagonalization of $h_{i j}=\epsilon_{i} \delta_{i j}+\Gamma_{i j}^{0}$. In the basis $\left\{v_{i}\right\}$ of the corresponding eigenstates, $\rho^{0}$ and thus $\tilde{G}^{0}$ are diagonal, so that it will be convenient to solve Eqs. (5) just in this basis. This is done by the standard iteration procedure. ${ }^{4}$

After adding and subtracting the term $\sum_{i j} \Gamma_{i j}^{0} a_{i}^{\dagger} a_{j}$, we transform the Hamiltonian (1) into this basis. Writing Eqs. (3) and (4) as

$$
\begin{aligned}
& \Gamma=\Gamma^{(2)}+\frac{1}{2} \Gamma^{(\rho)}+\frac{1}{4} \Gamma^{(\phi)}, \\
& \Delta=\frac{1}{2} \Delta^{(2)}+\frac{1}{2} \Delta^{(\rho)},
\end{aligned}
$$

its ground-state expectation value becomes

$$
E_{0}=\sum_{v} \epsilon_{v} \rho_{v v}+\sum_{v_{1} v_{2}} \rho_{v_{2} v_{1}}\left(\frac{1}{2} \Gamma_{v_{1} v_{2}}^{(2)}+\frac{1}{6} \Gamma_{v_{1} v_{2}}^{(\rho)}-\Gamma_{v_{1} v_{2}}^{0}\right)+\frac{1}{2} \sum_{v_{1} v_{2}} \phi_{v_{1} v_{2}}^{*} \Delta_{v_{1} v_{2}} .
$$


The term containing $\Gamma^{0}$ corrects for those contributions of $V^{(2)}$ and $V^{(3)}$ already contained in the singleparticle levels $\epsilon_{v}$. Working from the beginning in this representation, we would have obtained directly Eqs. (5). But the procedure sketched above gives more insight into the way double countings are to be avoided, and a definite prescription to calculate $\Gamma^{0}$. Expression (9) gives the correct contributions of the residual interaction to the energy. Starting with a Hamiltonian containing a single-particle potential, the absolute value of the ground-state energy cannot be calculated, because we cannot tell how much of the interaction defining this potential will be counted twice. But taking the same initial potential for a whole chain of nuclei, we are able to calculate energy differences, the unknown terms dropping out. The assumption is, of course, that the residual interaction yields a good approximation to $\delta \Gamma$, i.e., the change in the single-particle potential.

Once arrived at Eqs. (5) and (9) we take as singleparticle basis the eigenstates of a Woods-Saxon potential. $^{5}$

Deriving Eqs. (5) within the Green's-function formalism we see that in the definitions of $\Gamma$ and $\Delta$ there should appear an effective interaction rather than the bare one, accounting for higher-order contributions as shown by Migdal. ${ }^{6}$ The arguments concerning the difference between particle-particle (pp) and particle-hole (ph) interaction apply to the three-body force as well. Because of time-reversal symmetry the pph and phh matrix elements in the second terms of Eqs. (3) and (4) should be parametrized in the same way, whereas the third term in Eq. (3) is of ppp type and may be treated differently. Extending the argumentation of Klemt, Moszkowski, and Speth ${ }^{7}$ we antisymmetrize the three-body part in the pp but not in the ph channels. As a bonus this avoids the problem of the so-called "nuclear ferromagnetism" in systems without spin saturation, ${ }^{8}$ because all the critical contributions proportional to the spin densities arise from exchange terms of the interaction matrix elements. ${ }^{9}$ In this connection we note that, regarding the form of the $\rho$ dependence, our prescription is equivalent to that of Bäckman, Jackson, and Speth, ${ }^{10}$ whereas the parametrization of Waroquier, Heyde, and Wenes ${ }^{11}$ leads to a partial suppression of the exchange terms, i.e., no proper antisymmetrization as well.

It is known ${ }^{4}$ that any density dependence in some sense corresponds to many-body parts in the effective interaction. But there is one important thing to note: In contrast to the Hartree-Fock case, in the presence of pairing a three-body force gives rise to contributions which could only be interpreted in terms of a twoparticle interaction, if one allowed for a generalized density dependence including the anomalous density $\phi$. With an interaction like, e.g., that of Ref. 10, Dechargé and Gogny ${ }^{12}$ or Dobaczewski, Flocard, and Treiner ${ }^{13}$ the Green's-function formalism would not give the term $\frac{1}{4} \Gamma^{(\phi)}$ in Eq. (7), which is the essential one producing the odd-even staggering of the charge radii (and the kink at magic numbers) as has been explained in detail in Ref. 1.

For the two-body part of the effective interaction we make an Ansatz like that of Migdal, but without density interpolation, because we assume all the density dependence to be produced by $V^{(3)}$. In the ph channel we write

$$
F^{\mathrm{ph}}=C_{0} f \delta\left(\mathbf{r}_{1}-\mathbf{r}_{2}\right)
$$

where $f$ is different for like and for unlike particles. In terms of the more familiar $f_{0}, f_{0}^{\prime}$ we have

$$
f_{l}=f_{0}+f_{0}^{\prime}, f_{u}=f_{0}-f_{0}^{\prime}
$$

For the pp (pairing) force (acting only between like par-

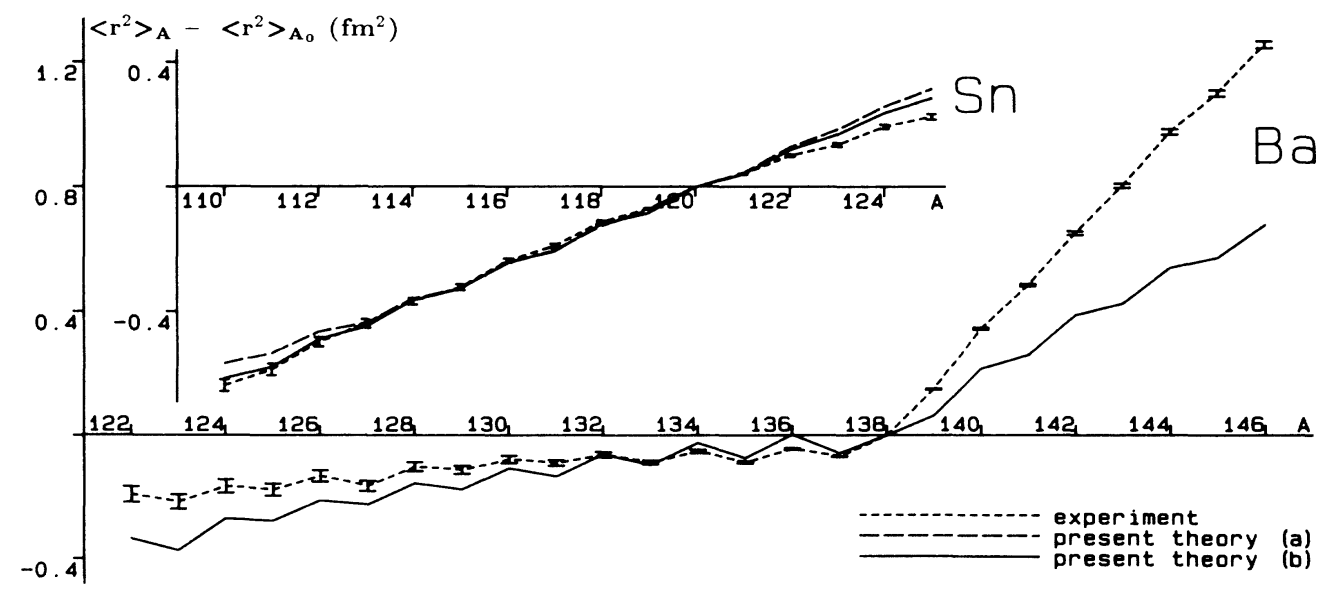

FIG. 1. Relative mean-square charge radii for the $\mathrm{Sn}$ and $\mathrm{Ba}$ isotopic chains. Experimental values taken from Ref. 16; present theory (a) results from Ref. 1; present theory (b) this work. 


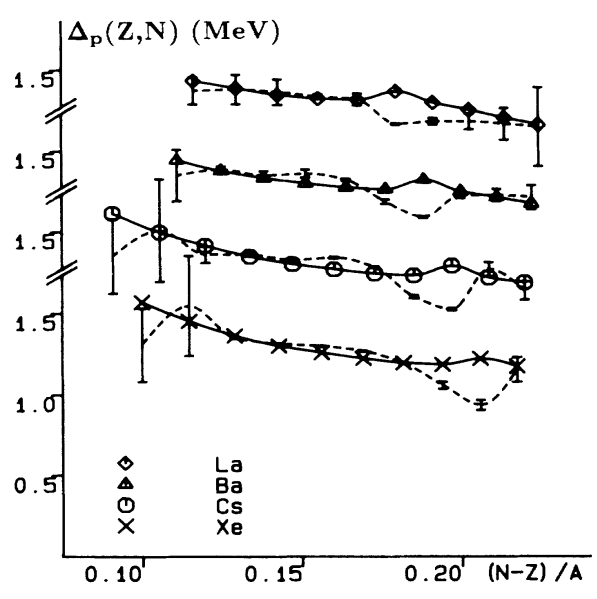

FIG. 2. Proton pairing energies defined according to Ref. 2. The lines are drawn to guide the eye. Dashed lines: experimental values calculated from Ref. 17; solid lines: present work.

ticles) we have $e^{1,14}$

$$
F^{\mathrm{pp}}=C_{0} L \delta\left(\mathbf{r}_{1}-\mathbf{r}_{2}\right) \frac{1}{2}\left[1-P_{\sigma}(1,2)\right] .
$$

$L$ is taken slightly different for proton-proton and neutron-neutron interaction, because in heavy nuclei with $N \neq Z$ the configuration spaces for protons and neutrons are generally not equivalent.

For the three-body part we take a $\delta$ interaction with different strength factors $C_{0} V_{3}^{(\rho)}$ and $C_{0} V_{3}^{(\rho)}$ in the pph and ppp channel, respectively, the superscripts corresponding to those in Eqs. (7) and (8). We again distinguish between interaction of like or unlike particles in each of these channels:

$$
V_{(3)}^{(a)}= \begin{cases}V_{l}^{(\alpha)}, \pi \pi \pi \text { or } v v v \text { interaction, } \\ V_{u}^{(\alpha)}, \pi \pi v \text { or } \pi v v \text { interaction, }\end{cases}
$$

where $\alpha=\rho, \phi$. In Ref. 1 only the $\phi$-dependent contributions of $V^{(3)}$, i.e., the last term in Eq. (3), were taken into account, whereas the other ones depending on the normal density $\rho$ were considered to be already well approximated by the fixed density interpolation of the twobody force used. Then density changes enter only linearly into Eqs. (7) and (8).

In the present calculations all terms originating from
TABLE I. Parameters of the effective interaction. The units of $V^{(\rho)}$ and $V^{(\phi)}$ are cubed femtometers, the other parameters are dimensionless. $C_{0}=386 \mathrm{MeV} \mathrm{fm}^{3}$.

\begin{tabular}{ccccccccc}
\hline \hline & $f_{l}$ & $f_{u}$ & $L_{p}$ & $L_{n}$ & $V_{l}^{(\rho)}$ & $V_{u}^{(\rho)}$ & $V_{l}^{(\phi)}$ & $V_{u}^{(\phi)}$ \\
\hline Sn & -0.60 & -2.45 & -1.90 & -1.80 & 10.0 & 16.0 & 12.0 & 31.0 \\
Ba & -0.50 & -2.40 & -2.13 & -1.81 & 10.0 & 16.0 & 19.0 & 58.0 \\
\hline \hline
\end{tabular}

the three-body component are treated self-consistently, giving rise to an additional quadratic dependence of $\Gamma$ and $\Delta$ on variations of $\rho$. This yields on the one hand the essential rearrangement term $\frac{1}{6} \sum_{v_{1} v_{2}} \rho_{v_{2} v_{1}} \Gamma_{v_{1} v_{2}}^{(\rho)}$ in Eq. (9), without which even binding-energy differences cannot sensibly be calculated. On the other hand, $\Gamma^{(\rho)}$, depending on $\rho^{2}$ and contributing to Eq. (7) with the opposite sign as compared to $\Gamma^{(2)}$, produces the negative curvature of the radii as a function of neutron number, experimentally observed, ${ }^{15,16}$ e.g., in the tin isotopes (Fig. 1). As mentioned above, the odd-even staggering as well as the kink at the closed shell exhibited by our Ba results is produced by $\Gamma^{(\phi)}$. We want to stress that these are no deformation effects, since we are working entirely within a spherical model [all densities entering Eqs. (5) being explicitly rotationally invariant ${ }^{12}$ ].

The treatment of $\Delta^{(\rho)}$ is of little influence on the charge radius, but it significantly affects the pairing energies. When adding neutrons, this contribution to the pair potential, also originating from the repulsive threebody component of our interaction, gets stronger, which is equivalent to a reduction of the effective pairing interaction strength. This, in our opinion, is the cause of the isotopic effect in the pairing energies observed by Vogel, Johnson, and Hansen. ${ }^{2}$ In particular, we get the decrease of the proton pairing with increasing neutron number (Fig. 2) without the invocation of protonneutron pairing. ${ }^{18}$

We calculate the quantity $\Delta p(Z, N)$ defined according to Ref. 2 from Eq. (9) evaluated for different proton and neutron numbers with ${ }^{134} \mathrm{Ba}$ as reference nucleus. The interaction and potential parameters are given in Tables I and II, respectively. All single-particle states up to three major shells above the Fermi energy are included in the configuration space. In the $\mathrm{Ba}$ region the level spacings around $\epsilon_{\mathrm{F}}$ are adjusted according to the experi-

TABLE II. Parameters of the Woods-Saxon potential defined according to Ref. 5. Except

\begin{tabular}{|c|c|c|c|c|c|c|c|c|c|c|c|c|c|c|}
\hline & \multicolumn{2}{|c|}{$\begin{array}{c}V_{0} \\
(\mathrm{MeV})\end{array}$} & \multicolumn{2}{|c|}{$\begin{array}{c}R_{0} \\
(\mathrm{fm})\end{array}$} & \multicolumn{2}{|c|}{$\begin{array}{c}\alpha_{0} \\
(\mathrm{fm})\end{array}$} & \multicolumn{2}{|c|}{$\lambda$} & \multicolumn{2}{|c|}{$\begin{array}{l}R_{L S} \\
(\mathrm{fm})\end{array}$} & \multicolumn{2}{|c|}{$\begin{array}{c}\alpha_{L S} \\
(\mathrm{fm})\end{array}$} & \multirow{2}{*}{$\begin{array}{c}R_{C} \\
(\mathrm{fm})\end{array}$} & \multirow{2}{*}{$\begin{array}{c}b \\
(\mathrm{fm})\end{array}$} \\
\hline & $p$ & $n$ & $p$ & $n$ & $p$ & $n$ & $p$ & $n$ & $p$ & $n$ & $p$ & $n$ & & \\
\hline $\mathrm{Sn}$ & 52.9 & 43.5 & 6.33 & 6.33 & 0.67 & 0.67 & 32 & 32 & 6.33 & 6.33 & 0.67 & 0.67 & 6.33 & 2.236 \\
\hline $\mathrm{Ba}$ & 57.5 & 45.8 & 6.63 & 6.40 & 0.79 & 0.66 & 27 & 32 & 6.40 & 6.19 & 0.59 & 0.64 & 6.58 & 2.277 \\
\hline
\end{tabular}
for $V_{0}$ the $\mathrm{Ba}$ values are taken from Ref. 19; $b$ is the oscillator length. 
mental values quoted in Ref. 19, in Sn the $\frac{11}{2}^{-}$(intruder) state is shifted down by $1.3 \mathrm{MeV}$ to obtain the correct level ordering.

Figure 2 shows our results for $\Delta_{p}(Z, N)$ for some isotopic chains around $\mathrm{Ba}$ compared to the experimental values. ${ }^{17}$ Apart from shell effects the decreasing trend is reproduced very well. Note that all these results are obtained without any parameter variation. The slightly different parameters for $\mathrm{Sn}$ can be tolerated in view of the extremely simple form of our interaction.

Thus we conclude that with a suitable three-body part in the effective interaction one is easily able to explain at the same time general properties of nuclear charge radii and pairing energies.

This work was supported by the Deutsche Forschungsgemeinschaft, No. Za-119. We thank Dr. R. Stapel for many valuable discussions. Numerical calculations have been performed at the Regionales Rechenzentrum für Niedersachsen (RRZN).

${ }^{1}$ D. Zawischa, U. Regge, and R. Stapel, Phys. Lett. B 185, 299 (1987)

${ }^{2}$ P. Vogel, B. Johnson, and P. G. Hansen, Phys. Lett. 139B,
227 (1984).

${ }^{3}$ L. P. Gorkov, Zh. Eksp. Teor. Fiz. 34, 505 (1958) [Sov. Phys. JETP 7, 347 (1958)].

${ }^{4} \mathrm{P}$. Ring and P. Schuck, The Nuclear Many-Body Problem (Springer-Verlag, Berlin, 1980).

${ }^{5}$ J. Speth, E. Werner, and W. Wild, Phys. Rep. 33C, 127 (1977).

${ }^{6}$ A. B. Migdal, Theory of Finite Fermi Systems and Application to Atomic Nuclei (Wiley, New York, 1967).

${ }^{7}$ V. Klemt, S. A. Moszkowski, and J. Speth, Phys. Rev. C 14, 302 (1976).

${ }^{8}$ B. D. Chang, Phys. Lett. 56B, 205 (1975).

${ }^{9}$ D. Vautherin and D. M. Brink, Phys. Rev. C 5, 626 (1972).

${ }^{10}$ S.-O. Bäckman, A. D. Jackson, and J. Speth, Phys. Lett. 56B, 209 (1975).

${ }^{11} \mathrm{M}$. Waroquier, K. Heyde, and G. Wenes, Nucl. Phys. A404, 269 (1983).

12 J. Dechargé and D. Gogny, Phys. Rev. C 21, 1568 (1980).

13 J. Dobaczewski, H. Flocard, and J. Treiner, Nucl. Phys. A422, 103 (1984).

${ }^{14}$ D. Zawischa, Phys. Lett. 155B, 309 (1985).

${ }^{15}$ P. Aufmuth, K. Heilig, and A. Steudel, At. Data Nucl. Data Tables 37, 455 (1987), and references therein.

${ }^{16}$ K. Heilig, Hyperfine Interact. 24-26, 349 (1985).

${ }^{17}$ A. H. Wapstra and G. Audi, Nucl. Phys. A432, 1 (1985).

${ }^{18}$ A. S. Jensen and A. Miranda, Nucl. Phys. A449, 331 (1986).

${ }^{19} \mathrm{C}$. Conci, thesis, Jülich University, 1984 (unpublished). 